\section{P246 THE ROLE OF PRIOR SYPHILIS IN THE CLINICAL PRESENTATION OF THIS DISEASE AMONG ADULTS FROM STI CLINICS IN PERU}

${ }^{1} \mathrm{E}$ Reyes Diaz*, 1,2K Konda, 'S Vargas, ${ }^{1} \mathrm{G}$ Calvo, ${ }^{1} \mathrm{C}$ Cáceres, ${ }^{2} \mathrm{~K}$ Klausner. ${ }^{1} \mathrm{Centro}$ de Invesstigación Interdisciplinaria en Sexualidad, SIDA y Sociedad (CIISSS), UPCH, Lima, Perú; 2University of California, Los Angeles (UCLA), Los Angeles, USA

\subsection{6/sextrans-2021-sti.327}

Background Individuals with prior syphilis are believed to be less symptomatic at subsequent infections. We present preliminary data using baseline information from the PICASSO cohort among people with active syphilis to compare the clinical presentations of individuals with and without prior Treponema Pallidum (TP) infection.

Methodology We included people with active early syphilis who fulfilled one of the following criteria: 1) current RPR titer of 1:8 plus a four-fold rise in RPR results within a year or 2) a new lesion with a positive TP-PCR DNA result from lesion swab. Syphilis naive (first infection) was defined as having a previous non-reactive TP-rapid test (TP-RT) within a year, and reinfection as having a prior reactive TP-RT or a prior $\mathrm{RPR} \geq 1: 8$ within a year. Chi2-square tests were used to examine associations.

Results Among the 91 men and transgender women with early syphilis: there were 10 primary (11\%), 10 secondary (11\%) and 71 latent or asymptomatic (78\%) infections. Penile painless ulcers were the most common presentation of primary syphilis $(80 \%, 8 / 10)$. All secondary syphilis cases had cutaneous rash. Thirty-six (40\%) were syphilis naive, and $62(68 \%)$ were HIV negative. Of the latter, 48/62 (77\%) were on PrEP. Symptomatic syphilis was more frequent in the syphilis naive group (39\% vs 11\%, chi2 p=0.002). Among $62 \mathrm{HIV-negative}$ participants, symptomatic syphilis was less frequent among PrEP users $(15 \%$ vs $43 \%$, chi2 $\mathrm{p}=0.022)$. Prior syphilis was also higher among PrEP users but no statistically significant (54\% vs 27\%, chi2 $\mathrm{p}=0.063$ ). No significant difference was found by HIV status.

Conclusion Prior TP infection impacts clinical presentation during subsequent infections, reducing the likelihood of diagnosing primary or secondary syphilis. The finding of less frequent symptomatic cases among PrEP users may be related to higher prior syphilis among them in our study.

\section{P250 POTENTIAL INTERACTIONS BETWEEN THE PATHWAYS TO DIAGNOSIS OF HIV/STI AND HIV SELF-TESTING: A QUALITATIVE STUDY OF GAY MEN IN SINGAPORE}

${ }^{1} \mathrm{R}$ Tan* ${ }^{2}{ }^{2}$ Chan, ${ }^{2} \mathrm{M}$ Ibrahim, ${ }^{2} \mathrm{~L}$ Ho, ${ }^{2} \mathrm{O}$ Lim, ${ }^{2} \mathrm{~B}$ Choong, ${ }^{3} \mathrm{M}$ Chio, ${ }^{2} \mathrm{M}$ Chen, ${ }^{4} \mathrm{C}$ Wong ${ }^{1}$ Saw Swee Hock School of Public Health, National University of Singapore, Singapore, Singapore; ${ }^{2}$ National Centre for Infectious Diseases, Singapore, Singapore; ${ }^{3}$ Department of STI Control (DSC) Clinic, National Skin Centre, Singapore, Singapore; ${ }^{4}$ Global Health and Population Research, FHI 360, Durham, USA

\subsection{6/sextrans-2021-sti.328}

Background This study draws on qualitative insights on the barriers and facilitators to HIV testing, as well as perceptions of HIV self-testing (HIVST), to propose a framework to understand the benefits, but also potential knock-on implications of introducing HIVST in the context of other STI testing.

Methods We conducted semi-structured, in-depth interviews with 30 gay, bisexual and other men who have sex with men (GBMSM) aged 18 and 39 years old in Singapore. Interview topics included barriers and facilitators to HIV and other STI testing, as well as perceptions of HIVST. Interviews were audiorecorded, transcribed, coded, and analysed using thematic analysis. Results For HIV testing, participants cited the perceived risk of acquiring, susceptibility to, and symptoms of HIV as internal motivators, while social influence and accessibility of HIV testing services were external motivators. For STI testing, perceived symptoms and partner notification of STI were reported as an internal and external motivator, respectively. Availability of bundle tests, starting a new relationship, and instances of mandatory testing motivated both simultaneous HIV and other STI testing. The fear of a positive diagnosis and lack of confidentiality were cited as internal and external barriers to HIV testing, respectively, while low perceived severity of other STI and the cost of STI tests were cited as internal and external barriers to other STI testing, respectively. We identified pathways to HIV and other STI testing and discussed how the introduction of HIVST may reduce opportunities for other STI testing.

Conclusions The findings of this study suggest that introducing HIVST might weaken linkages to other STI testing if alternative strategies of promoting other STI testing are not simultaneously implemented. We recommend that future interventions address both the risks of HIV and other STI simultaneously, and that structural interventions promoting HIV and other STI prevention be balanced accordingly.

\section{P254 THE LASTING IMPACT OF THE COVID 19 PANDEMIC ON SEXUAL HEALTH CARE IN A METROPOLITAN REGION}

${ }^{1} \mathrm{~A}$ Trolard*, ${ }^{2} \mathrm{~L}$ Weingarten, ${ }^{1} \mathrm{~J}$ Cherabie, ${ }^{1} \mathrm{H}$ Reno. ${ }^{1}$ Washington University, Saint Louis, USA; ${ }^{2}$ Saint Louis County Health Department, Saint Louis, USA

\subsection{6/sextrans-2021-sti.329}

Background In the US, health policies accompanying the COVID-19 pandemic led to restricted healthcare access and decreased clinical services. Low public health funding was a barrier to accessing sexual healthcare before the pandemic and intensified during restrictions. Surveillance of the sexual healthcare landscape can inform program development to improve sexual health.

Methods We collected information from clinics offering STI testing in the St. Louis region, Missouri, USA twice during 2020: in the spring during the first pandemic lockdown and in the fall as restrictions eased. Clinics were interviewed about sexual health services and categorized into three categories: open fully with no changes; modified if hours open and/or the ability to accept walk-ins were reduced; or fully closed. We also collected chlamydia rates by zip code and categorized zip codes as low (0-174 cases per 100,000); medium (175653); or high (654-1291) prevalence.

Results Of the 112 clinics, 47 are federally qualified health centers (serve the un-and underinsured); 16 were local public health authorities; and the remaining 49 include community, school, and private settings. In the fall, $29 \%$ of clinics were open, 55\% modified, 5\% closed compared with spring when $7 \%$ of clinics were open, $63 \%$ were modified, $17 \%$ were closed. There was a $450 \%$ and $200 \%$ increase in the number of clinics that were open in high and medium prevalence zip codes respectively in the fall compared to the spring. Fortynine clinics remain modified or closed in medium and high prevalence zip codes. 
Discussion The COVID pandemic has a significant effect on sexual health care in a metropolitan region of the US; that effect is lasting, especially in areas of medium and high chlamydia rates. Health systems must consider how the pandemic has impacted care provided for all major public health problems, including STIs/HIV or risk worsening sexual health.

\section{P255 TROUBLING SILENCES: REFLECTING ON FINDINGS FROM AN INTEGRATIVE REVIEW ON ACCESS TO PREP}

${ }^{1} T$ Trombetta*, ${ }^{2}$ J Benito Tovillo, ${ }^{2} \mathrm{~V}$ Caine. ${ }^{1}$ Go Freddie, Edmonton, Canada; ${ }^{2}$ University of Alberta, Canada

10.1136/sextrans-2021-sti.330

Background In 2012, pre-exposure prophylaxis (PrEP) was approved as an HIV-prevention intervention. PrEP is a highly effective strategy for reducing the risk of HIV acquisition, particularly in populations at high risk of contracting the virus. In an integrative review of the literature, we explored barriers and facilitators to accessing PrEP and outlined potential interventions to mitigate access. In the integrative review, 48 studies were included. We also extracted data that provided information on potential interventions and recommendations that stakeholders and decision-makers can utilize to advance practice guidelines and health policies that will improve PrEP access among high-risk populations. In this paper, we reflect on the review findings and contemplate the silences that became visible when looking across all studies.

Methods We engaged in the process of reflexivity as we looked across the included studies. Throughout this process, we made notes, engaged in conversations, and consulted with others who work in the field. Results/

Conclusion We noted three significant areas of silence. One was a lack of intersectional analysis, which considers multiple minority-stress factors acting simultaneously. With little understanding of the complexities impacting understudied populations' intersectionalities, stakeholders and decision-makers lack not only formative contextual research, but also any effective implementable measure to increase PrEP uptake. The studies analyzed showed a lack of community-participatory research practice. At large, the studies found did not explore, nor perceive, communities at HIV risk as agents of their own health. Neither did they represent these communities as capable stakeholders and decision-makers in matters regarding sexual behavior and harm reduction. Peer-support involvement in public health measures to improve PrEP access has been scarce. Despite social connections and relationships representing efficient methods for PrEP awareness, education, and stigma reduction, peer involvement remains mostly unexplored in the literature in relation to PrEP access.

\section{P256 IDENTIFYING STI RISK GROUPS AMONG HETEROSEXUALS IN A COHORT STUDY BASED ON BEHAVIOURAL AND PSYCHOLOGICAL CHARACTERISTICS DURING THE COVID-19 PANDEMIC}

${ }^{1} \mathrm{D}$ van Wees ${ }^{*},{ }^{2} \mathrm{~N}$ Godijk, ${ }^{3} \mathrm{C}$ den Daas, ${ }^{2} \mathrm{M}$ Kretzschmar, ${ }^{1} \mathrm{~J}$ Heijne. ${ }^{1}$ Center for Infectious Diseases Control, National Institute for Public Health and the Environment, Bilthoven, The Netherlands; ${ }^{2}$ Julius Center for Health Sciences and Primary Care, University Medical Center Utrecht, Utrecht, The Netherlands; ${ }^{3}$ Aberdeen Health Psychology Group, Institute of Applied Health Sciences, Aberdeen, UK

10.1136/sextrans-2021-sti.331
Measures to reduce Coronavirus disease (COVID-19) transmission, including physical distancing, and downscaling of sexual health care impact behaviour and sexual health. We aimed to examine the impact of COVID-19 measures on sexual behaviour, and to characterize heterosexuals who were at high risk of acquiring sexually transmitted infection (STI) during the pandemic. A longitudinal cohort study (20162020) was conducted among Dutch heterosexual males and females aged 21-28 years in 2020. We used data on behavioural and psychological characteristics from: pre-lockdown (June-August 2019), lockdown (March-May 2020), and postlockdown (June-August 2020). Behaviour change was compared between subgroups identified with latent class analysis. Four latent classes were identified $(\mathrm{n}=239)$. Individuals in class 1 (48\% of study population) and $2(36 \%)$ were at low risk of acquiring STI pre-lockdown, during, and post-lockdown, and reported mostly steady partnerships. Individuals in class $3(9 \%)$ and $4(7 \%)$ reported multiple casual partners pre-lockdown, and class 4 continued having multiple partners during lockdown $(56 \%$ reported same/increased partner numbers compared to pre-lockdown versus $18 \%$ in class 3 ). Class 4 was characterized by less condom use, lower health goals, less positive STI/COVID-19 prevention attitudes, and higher impulsiveness compared to class 3. Post-lockdown, $36 \%$ in class 3 and $42 \%$ in class 4 reported same/increased partner numbers compared to pre-lockdown. Of individuals who wanted an STI test during or post-lockdown in class $3(57 \%)$ and $4(75 \%), 62 \%$ and $56 \%$ respectively did not get tested, mainly because they were not able to get an appointment. STI risk during the COVID-19 pandemic was low in most heterosexuals, but specific subgroups engaged in high-risk behaviour during lockdown and post-lockdown. During and after the COVID-19 pandemic, impulsive individuals with low health goals and less positive infection prevention attitudes should be prioritized for STI testing, and targeted with behavioural interventions tailored to these psychological characteristics.

\section{P257 ONLINE PARTNER SEEKING AS A SOCIAL PRACTICE: FINDINGS TO DEVELOP THE FOURTH NATIONAL SURVEY OF SEXUAL ATTITUDES AND LIFESTYLES}

${ }^{1} \mathrm{D}$ Reid*, ${ }^{1} \mathrm{C}$ Bonell, ${ }^{2} \mathrm{R}$ Lewis, ${ }^{3} \mathrm{~B}$ Hogan, ${ }^{2} \mathrm{~K}$ Mitchell, ${ }^{2} \mathrm{R}$ Bosó Pérez, ${ }^{4} \mathrm{~J}$ Gibbs, ${ }^{5} \mathrm{C}$ Smith, ${ }^{6} \mathrm{~F}$ Attwood, ${ }^{4} \mathrm{C}$ Mercer, ${ }^{4} \mathrm{P}$ Sonnenberg, ${ }^{1} \mathrm{~W}$ Macdowall, the Nastsal-4 Team. ${ }^{1}$ Department of Public Health, Environments and Society, London School of Hygiene and Tropical Medicine, London, UK; ${ }^{2} \mathrm{MRC/CSO}$ Social and Public Health Sciences Unit, University of Glasgow, Glasgow, UK; ${ }^{3}$ Oxford Internet Institute, University of Oxford, Oxford, UK; ${ }^{4}$ Institute for Global Health, University College London, London, UK; ${ }^{5}$ Northumbria University, Newcastle, UK; ${ }^{6}$ Independent Scholar, UK

\subsection{6/sextrans-2021-sti.332}

Background Rapid development and uptake of digital technologies have influenced sexual lives. As part of development research for the decennial British National Survey of Sexual Attitudes and Lifestyles (Natsal-4), we aimed to understand the practices of adults in Britain using digital technologies to meet sexual and romantic partners.

Methods We conducted 40 semi-structured interviews with adults in Britain on the role digital technologies played in their sexual lives. Here we draw on the accounts of 22 of those who had direct experience of online partner seeking. Informed by Social Practice Theory, we developed thematic 